\title{
GLAD!
}

Revue sur le langage, le genre, les sexualités

De la justesse de l'interprétation à la justice herméneutique : quelle(s) direction(s) pour un tournant féministe de l'herméneutique?

Right Interpretation and Hermeneutical Justice: What Directions for a Feminist Turn in Hermeneutics?

\section{Axelle Cressens}

\section{OpenEdition}

Journals

Édition électronique

URL : http://journals.openedition.org/glad/1963

DOI : 10.4000/glad.1963

ISSN : 2551-0819

Éditeur

Association GSL

\section{Référence électronique}

Axelle Cressens, «De la justesse de l'interprétation à la justice herméneutique : quelle(s) direction(s)

pour un tournant féministe de l'herméneutique? », GLAD! [En ligne], 09 | 2020, mis en ligne le 20 décembre 2020, consulté le 20 janvier 2021. URL : http://journals.openedition.org/glad/1963 ; DOI : https://doi.org/10.4000/glad.1963

Ce document a été généré automatiquement le 20 janvier 2021.

\section{(c) $($ ) $\odot \ominus$}

La revue GLAD! est mise à disposition selon les termes de la Licence Creative Commons Attribution Pas d'Utilisation Commerciale - Pas de Modification 4.0 International. 


\title{
De la justesse de l'interprétation à la justice herméneutique : quelle(s) direction(s) pour un tournant féministe de l'herméneutique?
}

\author{
Right Interpretation and Hermeneutical Justice: What Directions for a Feminist \\ Turn in Hermeneutics?
}

Axelle Cressens

1 Le présent article ${ }^{1}$ est une tentative pour explorer un territoire jusqu'ici peu fréquenté et encore moins revendiqué par les théoriciennes et les chercheuses féministes mais qui présente un intérêt indéniable quant aux études féministes orientées vers les problématiques du langage et de l'interprétation, ou encore qui questionnent nos rapports aux traditions de textes et de pensée: celui de l'herméneutique. Ayant longtemps été développée sous la forme d'une méthodologie pour la compréhension et l'interprétation juste des textes, l'herméneutique constitue aujourd'hui une approche théorique et pratique plus large, se définissant à la fois comme un courant de pensée, une épistémologie des sciences humaines, ou encore une dimension indissociable de l'expérience, celle-ci comprise comme élaboration continuelle de sens. Or, on peut se demander dans quelle mesure une telle approche peut dialoguer avec le champ du féminisme et plus généralement du politique, voire faire l'objet d'une appropriation féministe. Cette question sera déclinée ici selon un objectif qui est double: premièrement, revenir sur quelques interprétations que des autrices féministes ont consacrées à la pensée herméneutique dans les années 1980-1990 et revisiter leurs questionnements principaux ; deuxièmement - et indépendamment de cette tradition herméneutique restée coupée aussi bien des expériences et productions des femmes que des problématiques féministes et de genre -, s'interroger sur les enjeux et la pertinence d'une herméneutique féministe.

2 Né d'échanges autour de la traductologie et de la traduction féministes - le thème de ce numéro et un des enjeux importants du projet FELiCiTE -, mon travail constitue 
cependant une démarche dans laquelle ces thématiques resteront excentrées. Il n'en est pas moins qu'une recherche au croisement de l'herméneutique et du féminisme peut contribuer à mieux situer sinon à élargir le champ de la traductologie féministe, dont l'herméneutique féministe serait une approche adjacente et une théorie alliée. Dans cette étude, de tels apports et proximités se manifestent à plusieurs niveaux: d'abord, dans l'analyse des rapports que le féminisme a développés envers la théorie herméneutique et l'explicitation de leurs thématiques communes et leurs critiques souvent convergentes contre l'épistémologie positiviste ${ }^{2}$; aussi via l'interrogation critique du silence que la pensée herméneutique a maintenu à l'égard des femmes, la mise en cause de ses généralisations et de son universalisme gender blind ${ }^{3}$. En ce sens sont réactivées et réévaluées les connections et les divergences entre l'herméneutique et le féminisme en tant que traditions théoriques, mais aussi en tant que démarches critiques. Ensuite, à travers la question du potentiel herméneutique du féminisme (et ainsi de l'appropriation ${ }^{4}$ de l'approche herméneutique au sein du projet féministe) : aussi bien au sens où la perspective féministe, en investissant le paradigme de la compréhension, cherche à produire une vision du monde plus juste et plus réaliste ${ }^{5}$, qu'en tant que la pensée et la pratique féministes participent à l'élaboration de nouveaux langages et à l'émergence de nouveaux sens, via la mise en mots d'expériences réduites au silence et la récupération de voix rendues inaudibles. Ainsi, si le projet d'herméneutique féministe résonne avec le développement de l'approche féministe en traductologie et en traduction, il peut être fécond de penser ces appropriations féministes en parallèle, mais aussi en les croisant: en s'interrogeant donc sur la façon dont la déconstruction et la réactualisation féministes de l'herméneutique peuvent contribuer aux problématiques de la traductologie féministe, les deux champs de recherche ayant rapport à la production et à la transmission du sens. À la lumière du lien significatif entre l'herméneutique et la traduction (souligné notamment par Jane Wilhelm (2014), mais aussi dans la conception de la traductologie féministe adoptée par FELiCiTE'), le projet d'herméneutique féministe s'inscrit ainsi nécessairement dans le tournant féministe de la traductologie.

3 Maintenant, quels enjeux pour un tournant féministe de l'herméneutique? Dans les recherches récentes en sciences humaines et sociales, le genre dépasse de plus en plus le statut de simple outil ou d'angle d'approche particulier, pour devenir « une nouvelle logique du sens pour dire le réel» (Brugère 2014, cité par Paveau 2018:82). Cette nouvelle "herméneutique » à partir du genre n'invite-t-elle pas à revenir sur l'herméneutique elle-même qui s'est construite comme théorie et comme pratique dans l'oubli du genre? La question qui anime ma réflexion se formule donc ainsi : que pourrait être aujourd'hui une herméneutique féministe ? Ou en d'autres termes : quelle herméneutique pour la prise en compte des expériences des femmes et des opprimée's en général, des problématiques liées aux inégalités de genre et aux rapports de domination ? Ce projet vise donc à contribuer à une herméneutique qui tienne compte de la transversalité du genre dans nos traductions et interprétations de la réalité sociale, mais qui en même temps ne soit pas séparée de l'agentivité politique: susceptible ainsi de resituer l'interrogation herméneutique centrale qui porte sur la justesse de l'interprétation, en la prolongeant vers la question de la justice interprétative, ou herméneutique - une question également incontournable, me semble-t-il, pour la traductologie et la traduction féministes. Ces deux termes justesse/justice -, davantage qu'un simple jeu de mots, reflètent en réalité la direction que pourrait prendre le tournant féministe de l'herméneutique: sans abandonner 
l'impératif de la justesse (ou de la vérité), se donner l'impératif de la justice jusque-là absent, ou en tout cas trop marginal, dans la réflexion herméneutique.

Si l'herméneutique philosophique s'est construite dans l'oubli du genre, c'est qu'historiquement elle n'a pas su éviter, jusque dans ses développements récents (où le contraire semble être revendiqué à l'aide des concepts comme situation, historicité, etc.), d'opérer à partir d'une posture de «neutralité » et d'« universalité » philosophiques, sans notamment remettre en question les fondements androcentristes et eurocentristes de son approche. Comme le rappelle Diane Elam (1991: 356), le mot même herméneutique renvoie au masculin (et exclut le féminin), du fait de son lien à Hermès et à la fertilité masculine que celui-ci incarnait. L'autrice propose ainsi, afin de faire ressortir cette signification, de lire en anglais her-MEN-eutics avec men (hommes) souligné. Elam ne mentionne cependant pas que le même mot pourrait simplement être lu aussi comme HER-meneutics (à la manière de HER-story ${ }^{7}$ )!

Or, c'est d'emblée à la possibilité d'une herméneutique en tant que HER-meneutics (et non pas her-MEN-eutics) que s'intéresse ce projet de l'appropriation de l'herméneutique par le féminisme. Ce sont donc les conditions de cette appropriation, les difficultés aussi qui y sont liées, qui seront l'objet privilégié de mon étude, en m'amenant à poser des limites aussi bien à l'idée qu'il y aurait des ressemblances entre la tradition herméneutique et la pensée féministe, qu'à la question des «ressources» (Code 2003 : 2) que les féministes pourraient trouver dans cette tradition (question qui semble avoir encadré jusqu'ici la réflexion féministe sur l'herméneutique). L'intersection de l'herméneutique et du féminisme peut prendre une forme bien différente. L'idée ici n'est pas de revenir sur le courant herméneutique androcentriste afin d'y trouver un ancrage pour des idées féministes - les auteurs ${ }^{8}$ de ce courant sont loin d'être des alliés politiques du féminisme, ni même des «ami[s] silencieux des projets féministes » (Code $2003: 3)-$, mais d'esquisser un projet en vue de la construction d'une herméneutique féministe, celle-ci passant plutôt par l'explicitation de la dimension herméneutique au sein de la démarche féministe elle-même, sans nécessairement s'inscrire à l'horizon faussement universel de la tradition herméneutique.

L'expression « herméneutique féministe » n'est cependant pas nouvelle. Elle a sa place dès les années 1970 au sein des courants de la théologie féministe, où elle désigne la pratique de la lecture féministe des textes sacrés ${ }^{9}$. À ce titre, l'herméneutique y apparaît comme une approche pour la réinterprétation féministe du canon religieux patriarcal et, finalement, un outil de libération des femmes. Ce cas des théologies féministes reste toutefois spécifique: on ne mobilise l'herméneutique que dans le contexte de la révision de la tradition patriarcale dans l'exégèse théologique. Mais on peut déjà y reconnaître un des premiers cas de l'appropriation féministe de l'herméneutique. Ici, un nouveau potentiel de l'herméneutique apparaît grâce à ces travaux féministes, n'étant pas évident dans d'autres contextes (non-féministes) : c'est l'herméneutique entendue en termes de libération et donc déjà une herméneutique politique. Au-delà de ce cadre bien particulier, l'herméneutique féministe ne s'est pas imposée comme un projet aux côtés de la critique féministe du langage ou des épistémologies féministes.

Dans les pages qui suivent, il sera d'abord question de situer l'analyse féministe à l'égard de l'herméneutique traditionnelle. Pour ce faire, je mettrai en lumière quelques interprétations féministes du courant herméneutique, ainsi que le besoin de dépasser le 
cadre posé par ces études où le féminisme reste délimité par son rapport à la tradition herméneutique.

\section{Convergences entre l'herméneutique et le féminisme? Des épistémologies alternatives face au positivisme}

Il ne s'agit pas ici de retracer l'histoire du courant herméneutique. J'évoquerai très brièvement deux moments dans le développement théorique de cette pensée ${ }^{10}: a u x^{e}$ siècle, avec Martin Heidegger et Hans-Georg Gadamer, l'herméneutique a pris une orientation ontologique et existentielle, la revendiquant contre le statut d'épistémologie. Lorsque Gadamer (1982) écrit que la compréhension est « plus être que conscience", il veut insister sur le fait que ce n'est pas comme transparence à soi du savoir qu'elle doit être entendue, mais comme mode d'existence, celle-ci n'étant jamais complètement saisissable pour le sujet qui comprend. Plusieurs autrices féministes (Linda Martín Alcoff, Gloria Bowles, Eloise Buker, Lorraine Code, Georgia Warnke, parmi d'autres) ont néanmoins reconnu le potentiel épistémologique de l'herméneutique comme étant particulièrement intéressant pour le féminisme, et proposé de mobiliser ce potentiel en tant que fondement d'une connexion entre les deux courants. C'est que malgré ses réserves quant aux enjeux "épistémologiques " (visant plutôt une forme particulière de connaissance et d'épistémologie: le positivisme), l'herméneutique construit bien une certaine approche du savoir, une épistémologie. La lecture féministe de l'herméneutique passe par la réhabilitation de cette dimension épistémologique, sans pour autant perdre de vue les conséquences critiques (aussi bien épistémologiques que politiques) de sa théorie.

S'ouvre ainsi le second moment: la mise à distance de la sphère politique, notamment dans la pensée de Gadamer ${ }^{11}$. Plus généralement, les présupposés politiques ont souvent été passés sous silence chez les auteurs herméneutiques (à l'exception de Paul Ricœur et certains autres penseurs ${ }^{12}$ ), en tout cas leurs théorisations semblent être passées à côté du potentiel politique que pourraient avoir les questions du sens, de la compréhension, de l'interprétation, du dialogue, du langage, etc. L'oubli du politique indique aussi la non-reconnaissance des enjeux du pouvoir et de la domination qui traversent ces questions (Code $2003: 10,18$ ) : constat qui sera à l'origine d'une attitude critique de la part de féministes.

C'est autour de ces deux points que va se construire globalement l'analyse féministe de l'herméneutique, soulignant à la fois la proximité qui relie les questionnements herméneutiques et féministes en ce qui concerne les problématiques épistémologiques, et la rupture quant à leur lecture (ou non-lecture) politique. Cependant, malgré les limitations de l'herméneutique, la question de ses « usages " féministes semble être prédominante chez les autrices féministes. La communauté du féminisme et de l'herméneutique serait fondée dans la critique antipositiviste : la mise en question de la rationalité moderne, ainsi que de ses avatars que sont la subjectivité souveraine, la vérité objective, le savoir neutre. L'herméneutique et le féminisme ont proposé tous les deux des critiques majeures du logocentrisme ${ }^{13}$ auquel ils opposent des épistémologies alternatives ${ }^{14}$. En théorisant la compréhension en termes d'expérience et de praxis, l'herméneutique met en valeur le rôle des préjugés et des préconceptions dans la constitution des savoirs; elle prône la réflexivité et la compréhension de soi comme conditions indispensables du savoir qui se veut plus proche du monde de la vie. Enfin, 
elle replace le lieu de la vérité au sein du dialogue et de la communauté, où il ne s'agit plus de l'observation désintéressée, mais de la participation vivante et de la cohabitation langagière. Ces quelques points trouveront certainement écho auprès des épistémologies féministes. Sans passer ici en revue tous les points communs, je m'arrêterai sur quelques analyses féministes qui, tout en explicitant des arguments en faveur d'une coalition entre le féminisme et l'herméneutique, mettent également en lumière les divergences qui pourraient rendre une telle coalition difficile, voire lui faire clairement obstacle. J'essayerai ensuite d'argumenter en quoi considérer la théorie herméneutique comme une ressource féministe peut être problématique.

\section{Interprétations féministes de l'herméneutique : interroger l'utilité de l'herméneutique pour le féminisme}

11 Dans la mesure où la pensée herméneutique s'inscrit dans la tradition philosophique occidentale, il est ici utile de mobiliser des outils féministes élaborés dans le cadre de la critique et relecture du canon - un vaste projet de révision qui sous-tend les études féministes en histoire de la philosophie. Je m'inspirerai pour cela de l'analyse proposée par Charlotte Witt et Lisa Shapiro dans l'article de la Stanford Encyclopedia of Philosophy, "Feminist History of Philosophy" (Witt \& Shapiro 2014). En plus de faire l'état de différents modes d'interaction des philosophes féministes avec le canon philosophique, cet article met en évidence une tension caractéristique à mes yeux de l'approche féministe de la tradition patriarcale en général et qui est une tension herméneutique: entre, d'un côté, la démarche de l'appropriation du canon vu comme une ressource pour la pensée féministe et, de l'autre, la critique des méthodes traditionnelles de l'interprétation, qui vise l'élaboration de stratégies de lectures alternatives (" particulièrement sceptiques à l'égard du projet de l'appropriation ${ }^{15}$ ", ibid.) en vue de la déconstruction des biais sexistes et misogynes de la tradition.

Cette tension exprime deux tendances interprétatives dans les manières dont les autrices féministes ont formulé le rapport entre le féminisme et le courant herméneutique. La première approche se pose en termes d'usages possibles de la théorie herméneutique par le féminisme, ou plus généralement d'utilité de l'herméneutique pour les problématiques féministes (approche qui n'exclut pas pour autant une révision critique des présupposés de cette théorie). En soulignant les convergences entre l'herméneutique et le féminisme, notamment sur le niveau épistémologique, les autrices comme Bowles (1984) et Buker (1990), ou encore Warnke (1993), ont pu reconnaitre en l'herméneutique une partenaire solide de la critique féministe. Dans le même esprit d'utilité, Code (2003) et certain'e's contributrice teur·s à l'ouvrage collectif Feminist Interpretations of Gadamer envisagent la question des "ressources féministes » de l'herméneutique (notamment gadamérienne). Quant à la seconde approche - anti-appropriation -, elle s'avère beaucoup plus critique: l'herméneutique n'a pas grande chose à dire au féminisme, voire elle serait antiféministe puisqu'elle reste insensible à la question du genre et que son fondement même est bâti sur l'exclusion des femmes. C'est le cas par exemple des analyses d'Elam (1991) dans sa réponse à Buker et de Marie Fleming dont l'article propose une critique de la conception du dialogue chez Gadamer («Gadamer's Conversation : Does the Other Have a Say? ", in Code 2003). 
13 Les trois études féministes de l'herméneutique que je suggère ici pour l'analyse peuvent être inscrites dans l'une ou l'autre stratégie de l'interprétation. Tout en esquissant les arguments (pour ou contre) le rapprochement entre le féminisme et le courant herméneutique, ces travaux laissent en outre transparaître, souvent sans les mettre en avant, des potentialités herméneutiques propres au féminisme. C'est à la fois du point de vue de l'utilité (ou de la non-utilité) de l'herméneutique pour le féminisme et du potentiel herméneutique de la critique féministe elle-même que je propose maintenant de les examiner.

\section{Bowles et l'usage de l'herméneutique par le féminisme}

14 L'utilité de l'herméneutique pour le féminisme est interrogée par Bowles dans son article de 1984, «The Uses Of Hermeneutics For Feminist Scholarship » (« Les usages de l'herméneutique pour les études féministes »). Remarquons que l'herméneutique est ici entendue d'un côté en un sens très large, mais d'un autre assez réducteur puisque ramenée à un seul aspect: par ce mot, l'autrice désigne «une constellation de méthodologies qui se montrent critiques de l'objectivisme et du scientisme de la tradition mâle blanche» (p.186). Parmi ces «méthodologies», elle place certains courants de la pensée continentale de l'après-guerre (phénoménologie, herméneutique, post-structuralisme et déconstructivisme) qui se démarquent par leur rapport critique à la tradition.

15 Tout en se montrant consciente du danger de "l'usage abusif » de la pensée et des thématiques féministes par des philosophes mâles ${ }^{16}$, Bowles appelle à ne pas enfermer la théorie féministe dans une sorte de "ghetto intellectuel ", c'est-à-dire refuser de créer des connections avec les approches critiques non-féministes. Elle propose notamment de faire usage de ces «traditions hétérodoxes» comme stratégie pour promouvoir la pensée féministe en dehors du cercle des initiées, en contribuant par là à sa légitimation au sein de l'université ${ }^{17}$. Ainsi, ce que Bowles appelle «l'usage de l'herméneutique pour les études féministes » fait partie des «stratégies intellectuelles pour connecter nos critiques à d'autres qui questionnent également la tradition de pensée occidentale blanche»(p.186). C'est dans la communauté des critiques que résident pour l'autrice les "points d'intérêt communs » entre l'herméneutique et le féminisme, dans la mesure où pour les deux approches il s'agit de la mise en question de la tradition occidentale et ses présupposés logocentristes. Une mise en question qui, à travers l'herméneutique, ouvre une "porte » vers le féminisme: "Une voie vers la pensée féministe pour celleux qui se méfient de nous passe par la porte de l'herméneutique ou de la tradition interprétative dans laquelle on trouve une forme puissante de la critique du logocentrisme. » (ibid.). En suivant cette logique d'utilité, le fait de s'adresser à des courants de pensée non-féministes, voire androcentristes, n'est pas incompatible pour l'autrice avec la construction d'une théorie centrée sur les expériences des femmes, mais au contraire permet sa meilleure inscription dans le contexte intellectuel et théorique.

16 Cependant, malgré cette convergence des critiques que partagent l'herméneutique et le féminisme, Bowles met en garde contre le sexisme des théoriciens de l'herméneutique, qui s'exprime aussi bien par l'exclusion ou l'invisibilisation des femmes que par l'ignorance des travaux féministes, sinon par l'usage abusif qui en est fait. C'est là qu'apparaît l'insuffisance de la démarche critique de l'herméneutique : «Un nombre 
croissant d'[auteurs mâles blancs] ont pu faire la critique du logocentrisme; mais jusqu'à présent, aucun d'entre eux n'a pu analyser son propre sexisme. » (p. 188). En fin de compte, ces auteurs critiquent la tradition tout en se situant à l'intérieur de celle-ci et en partagent donc les biais sexistes et androcentristes. Des exemples "abondent ", ajoute Bowles dans une note (note 3 p. 186), où elle passe en revue quelques cas de ce qu'elle appelle " coupure entre la pensée et l'action dans des vies mâles ». Pour n'en citer que quelques-uns, des plus sidérants : le féminicide par Louis Althusser de son épouse, militante et sociologue Hélène Rytmann; les "assertion[s] "théorique[s]" " (Plaza 1978 : 95) de Michel Foucault à propos du viol ${ }^{18}$; ou encore le séminaire de Paul Feyerabend à la prestigieuse Eidgenössische Technische Hochschule de Zurich au début des années 1980, pour lequel il a invité 122 expert·es en trois ans et dont seulement huit étaient des femmes.

À l'écart de la tradition, les féministes seraient mieux placées pour la critiquer et pour élaborer des épistémologies alternatives (fondées sur l'expérience, la participation personnelle, le jugement, etc.). Pour cette raison aussi, selon l'épistémologie herméneutique (qui exige elle aussi la prise en compte des préjugés et des expériences personnelles), la pensée féministe, dans la mesure où elle met ces principes en œuvre, serait davantage susceptible de fournir une approche critique valable: ce qui laisse entendre chez Bowles une idée du potentiel herméneutique du féminisme.

La pensée traditionnelle prétend pouvoir sortir du cercle herméneutique/ interprétatif et parler de connaissances dites "libres de valeurs", "désintéressées », « objectives» et "éthiquement neutres». Ainsi, la tradition herméneutique/interprétative dit que tous les efforts pour nier la nature " circulaire ", « intéressée » et «évaluative » de la pensée sont conceptuellement confus ou malhonnêtes. La pensée féministe, précisément parce qu'elle reconnaît et affirme ses "préjugés", doit, du point de vue herméneutique, être considérée comme l'une des seules postures théoriques disponibles qui revendique l'intégrité intellectuelle et la sophistication. (p. 187)

\section{Buker : la « dialectique enrichissante » entre l'herméneutique et le féminisme?}

Buker dans son article «Feminist Social Theory and Hermeneutics: An Empowering Dialectics?» ("Théorie sociale féministe et herméneutique: une dialectique enrichissante ?») aborde le rapport entre l'herméneutique et le féminisme sous l'angle d'une dialectique possible et bénéfique pour les deux partis. Son interrogation, comme chez Bowles, est encadrée par l'idée de l'utilité de l'herméneutique pour la théorie féministe : Buker emploie le terme « utile » afin de désigner la contribution possible de l'herméneutique pour apporter une solution aux difficultés épistémologiques que rencontrent les féministes. Ainsi, sa thèse consiste à dire que "le féminisme peut devenir encore plus efficace dans la mesure où il prend en considération les idées de l'herméneutique philosophique pour organiser et articuler son récit de la réalité sociale et ses espoirs d'incarner une vie que les féministes peuvent appeler bonne » (Buker 1990 : 23). Exemple : le modèle épistémologique de l'herméneutique redéfinissant la recherche et l'objectivité scientifique de manière à y reconnaître le rôle explicite des valeurs sociales, pourrait faciliter pour la théorie féministe la tâche de concilier l'exigence d'une recherche centrée sur les femmes et basée sur leurs expériences personnelles avec la condition de la scientificité et de l'objectivité. Cependant, si l'approche de Bowles interrogeait principalement l'apport éventuel de l'herméneutique 
aux études féministes, celle de Buker présuppose une utilité réciproque. L'autrice suggère en effet que, tandis que l'herméneutique pourrait contribuer au développement d'une stratégie épistémologique pour la théorie sociale féministe, le féminisme pourrait donner à l'herméneutique une direction politique dont elle manque et ainsi l'aider à dépasser ses contradictions.

19 L'herméneutique (qu'elle renvoie le plus souvent à la théorie de Gadamer et, plus rarement, à celle de Ricœur) n'est envisagée par Buker que comme une " ressource » purement épistémologique et est disqualifiée quant aux questions du politique et du social. La portée épistémologique de l'herméneutique se résume pour elle avant tout dans la tâche de l'application ${ }^{19}$ et de l'interprétation qui s'ensuit - la tâche de trouver des moyens pour faire parler le texte au présent et à propos du présent, toujours en lien avec cette question : comment mener une vie bonne? Or, pour les théoriciennes féministes, il ne s'agit plus de "grands » textes ni de "grands» penseurs, mais de ce que Buker appelle le «texte d'une vie» (text of a life, p. 24) : des histoires personnelles des concernée's deviennent un moyen de l'analyse politique et éléments de la théorie sociale. Toutefois, c'est toujours la tâche de comprendre et d'interpréter ces histoires, ces "textes" de vies singulières, de les incorporer dans la théorie féministe, qui s'impose, et qui explique donc l'utilité de l'herméneutique.

Parmi les contributions possibles de l'herméneutique au féminisme, Buker mentionne la redéfinition de l'objectivité désormais basée sur la prise en compte du contexte social et historique; la prise en compte du rôle clé du langage dans la production et la reproduction de la réalité sociale (et donc dans la constitution de l'objet de la recherche féministe) ; l'importance de l'inclusion du soi de la chercheuse/du chercheur dans la recherche et l'exigence de la réflexivité; enfin le rapport à la tradition sous un mode qui ne serait pas exclusivement critique. Cependant, comme l'autrice le souligne à plusieurs reprises, l'herméneutique elle-même n'arrive pas à mettre en œuvre certains de ces points: elle manquerait ainsi de réflexivité pour ne pas avoir reconnu la dimension genrée du savoir et de l'expérience langagière.

21 Comme chez Bowles, l'échec de l'herméneutique de remplir ses propres engagements épistémologiques amène Buker à interroger le potentiel herméneutique du féminisme, à travers la dimension de la connaissance de soi. Elle oppose notamment à la perspective herméneutique traditionnelle une « herméneutique féministe » :

Les chercheuses et chercheurs qui travaillent dans des contextes androcentriques produisent de la recherche androcentrique - une recherche centrée sur les hommes. D'un point de vue herméneutique, ce n'est pas nécessairement une faute. Cela devient une faute dès lors que les chercheuses et chercheurs prétendent que la recherche représente à la fois les hommes et les femmes. D'un point de vue herméneutique féministe, cette revendication manque de connaissance de soi parce qu'elle ne connaît pas sa propre perspective genrée. C'est cette fausse prétention à l'universalité qu'une herméneutique féministe rejette. (p. 27, je souligne)

Ce point rejoint manifestement l'argument de Bowles - sur la réflexivité de l'approche féministe et sa capacité plus conséquente de tenir compte des préjugés (les siens comme ceux des autres). Enfin, dans un court paragraphe vers la fin du texte qui s'intitule «A feminist self-understanding: feminist hermeneutics» (p. 36-37), Buker définit l'herméneutique féministe en termes de compréhension de soi féministe. Ici, suivre l'herméneutique signifie célébrer l'inclusion de soi. Une herméneutique est féministe dans la mesure où elle inclut les femmes (le «soi») - non seulement les 
expériences et histoires personnelles des femmes, mais la pensée des femmes philosophes - dans la production du savoir et le travail de l'interprétation.

\section{Elam répond à Buker : le féminisme n'est pas la grâce salvatrice de I'herméneutique}

Le dernier texte qu'il s'agit d'analyser - celui d'Elam - est encore conditionné par cette question du rapport entre le courant herméneutique et le féminisme, seulement son approche de l'herméneutique s'avère beaucoup plus sceptique que chez les deux autrices précédentes. Son article est en fait une réponse à la contribution de Buker ${ }^{20}$, critique notamment de l'optimisme que cette dernière exprime quant à la coalition possible (rendue en termes de "dialectique enrichissante ») entre le féminisme et l'herméneutique. Aux yeux d'Elam, la faute de Buker serait de ne pas tenir compte de l'androcentrisme (Elam utilise le terme " phallocentrisme ») de l'herméneutique, et de faire travailler celle-ci dans des sens auxquels elle renonce ouvertement, en omettant notamment son refus de reconnaître la catégorie du genre et la différenciation sexuée. Or, souligne Elam, «le féminisme peut vouloir insister sur sa différence irréconciliable avec l'herméneutique»(p.350). Aux suggestions de Buker quant aux contributions réciproques de l'herméneutique et du féminisme, elle donne cette réponse: "Le féminisme n'est pas la grâce salvatrice de l'herméneutique et l'herméneutique ne répond pas aux préoccupations du féminisme » (ibid.).

Sous l'« herméneutique ", Elam entend ici quelque chose de beaucoup plus précis que Bowles et Buker. Elle désigne par là une branche de la philosophie heideggérienne qui a été développée par Gadamer et ses disciples, qu'elle définit comme sa branche « conservatrice » (en suivant ici la catégorisation de John Caputo (1987), par opposition à l'« herméneutique radicale » dont Caputo lui-même fait partie et où il range aussi la déconstruction de Jacques Derrida). C'est vers la déconstruction qu'Elam se tourne finalement, en affirmant que cette approche serait plus adéquate pour penser les problématiques de la différence des sexes, et aurait une relation plus positive avec le féminisme. Elle renvoie ainsi aux analyses de Derrida sur le féminin dans Éperons (1978) et à l'interprétation qu'en fait Caputo dans Radical Hermeneutics, en citant notamment la phrase de ce dernier, «[la] femme sonne le glas de l'herméneutique» (Caputo 1987: 157), pour insister encore une fois sur l'incompatibilité de l'herméneutique et du féminisme.

Le texte d'Elam présente une des premières critiques féministes radicales envers l'herméneutique philosophique. Sa conclusion proposant de se tourner vers la déconstruction pour son étude de la question du genre, telle qu'élaborée par Derrida dans Éperons, n'est cependant pas très satisfaisante ${ }^{21}$. Néanmoins, cette analyse reste utile quant à la mise en lumière de l'androcentrisme de l'herméneutique, en s'appliquant notamment à montrer le déni par la tradition herméneutique (et en particulier par sa branche gadamérienne) de la catégorie de genre. En somme, l'analyse d'Elam présuppose la nécessité du rejet par le féminisme de l'herméneutique traditionnelle, pour cause de son phallocentrisme, au profit d'un dialogue avec l'« herméneutique radicale » qu'est la déconstruction.

Bien que leurs manières d'aborder l'intersection entre le féminisme et l'herméneutique ne soient pas identiques, les trois autrices s'accordent autour d'un point principal: l'approche herméneutique, élaborée par des hommes et selon une perspective 
androcentriste, est porteuse d'un oubli essentiel, à savoir l'oubli du genre. En quoi cet oubli rendrait-il contradictoire la posture épistémologique de l'herméneutique?

\section{La théorie herméneutique gender blind : " situer » les savoirs pour une interprétation plus « juste »?}

Si l'on revient sur la tension formulée par Witt et Shapiro et évoquée plus haut, la question se pose de nouveau: quelle stratégie interprétative féministe face à la tradition herméneutique ? À la différence d'autres philosophies (dont celles d'Aristote ou de Kant), la pensée herméneutique n'associe pas ouvertement l'universel et le neutre au masculin, mais adopte une perspective gender blind. Code le résume ainsi dans le contexte de son analyse critique de l'herméneutique gadamérienne: il n'y a pas de "question féminine» (woman question) pour Gadamer (Code 2003: 2), en soulignant notamment l'absence dans son œuvre de propos misogynes ou de théorisations négatives du féminin. À défaut donc d'être incluse dans le "canon négatif » (Witt \& Shapiro 2014) des féministes - d'où peut-être la tentation de certaines autrices de voir en Gadamer un féministe silencieux ou « naissant» (nascently feminist, Alcoff $2003: 232$ ) - , la théorie gadamérienne (comme plus généralement la théorie herméneutique telle qu'elle s'est constituée au $\mathrm{xx}^{\mathrm{e}}$ siècle) n'en véhicule pas moins des biais implicites de genre, tels que l'androcentrisme.

La critique féministe qui pourrait correspondre à cette posture gender blind, consisterait selon Witt et Shapiro (2014) à " sonder les théories des philosophes canoniques afin de découvrir les préjugés sexistes qui se cachent dans leurs théories prétendument universelles", dans le cadre d'une stratégie qu'elles appellent "interprétation synoptique $»^{22}$ (synoptical interpretation) du canon. Si Gadamer, Ricœur et d'autres herméneuticiens ne sont pas des philosophes « canoniques » au même titre que Kant et Aristote, leurs textes et leurs théories (dans la mesure notamment où elles sont destinées à élaborer les outils mêmes de l'interprétation de la tradition) n'en participent pas moins à la construction et la perpétuation du canon. Celui-ci s'appuie sur « l'image de soi de la philosophie comme universelle et objective plutôt que comme particulière et partiale » (Witt \& Shapiro 2014). Bien qu'elle s'attaque elle-même à cette image, l'herméneutique ne prend pas en compte sa propre ignorance du genre (gender blindness), ce qui témoigne d'une défaillance dans sa démarche épistémologique vers un modèle « situé » du savoir.

L'épistémologie alternative que l'herméneutique oppose à l'objectivisme de la science, est basée sur le principe du " cercle herméneutique ", théorisé d'abord par Heidegger et repris par Gadamer comme fondement de sa théorie de l'expérience herméneutique : la connaissance ne constitue pas une trajectoire linéaire, mais relève d'un cercle, dans lequel nous sommes toujours déjà entrainée's, et qui rend impossible un savoir soidisant neutre, désintéressé, à distance. Nos préjugés précèdent en effet tout acte de comprendre, et notre expérience personnelle et historique prend part à ce que nous visons à connaître. Cela revient à accorder une place cruciale à la figure de l'interprète et à définir fondamentalement la rationalité herméneutique en termes de situation et de participation (Gadamer 1996). Or, cette rationalité soi-disant "située » ne l'est pas véritablement. Partant de la notion de situation concrète et de la figure de l'interprète, l'herméneutique laisse cependant totalement impensées les dimensions d'abord corporelle et matérielle, mais aussi politique de tout être-situé, et par conséquent ne va 
pas aussi loin qu'elle ne le prétend. Elle n'est ainsi pas en mesure de reconnaître les inégalités des situations herméneutiques et les différences de ses sujets liées au genre, à la race, à la classe, etc. : le fait que les sujets occupent différemment leur position de parole et d'interprétation. Le sujet dans la théorie herméneutique est posé comme prétendument neutre, mais en réalité il renvoie au masculin (et au blanc), et n'est pas en ce sens différent du sujet mâle ${ }^{23}$ et blanc de la tradition philosophique en général.

C'est donc un manque qui investit l'ensemble du modèle herméneutique de connaissance, lequel par conséquent pose problème, de ce fait la prétention de l'herméneutique à la vérité - à la justesse de l'interprétation - se trouve aussi problématique. Dans la mesure où il inclut la situation propre au sujet de l'expérience herméneutique, le savoir herméneutique prétend embrasser de plus près la réalité du monde que ne le fait le savoir objectiviste. Comme le souligne Code, « la compréhension herméneutique est moins définitive que le savoir empirique orthodoxal vise à l'être, mais en un certain sens "plus vraie" à l'égard des textes et des expériences auxquelles elle a affaire » (2003: 5). Or, cette prétention à la vérité, c'est-à-dire, à l'interprétation toujours plus adéquate se produisant dans une proximité toujours plus grande au monde est compromise lorsque l'herméneutique passe à côté des inégalités genrées, raciales ou de classe et les oppressions qui y sont liées.

31 En ce sens, une théorie féministe consciente de ces réalités de l'oppression et de l'inégalité a davantage réussi dans la tâche herméneutique que ne l'a fait l'herméneutique elle-même (c'est bien la conclusion à laquelle arrivent Bowles et Buker). Autrement dit, on ne peut pas atteindre la justesse dans l'interprétation sans engager une interrogation sur la justice rendue par l'interprétation et, plus largement, sur le projet d'un monde plus juste. C'est pourquoi la perspective épistémologique de l'herméneutique ne devrait pas être séparée de la perspective politique, et les questions du sens et de la vérité des problématiques de l'égalité et de la liberté. Une telle convergence de perspectives pourrait constituer un élément fondateur d'une herméneutique féministe, plus particulièrement à travers la question de la justice (et de l'injustice) herméneutique sur laquelle je reviendrai dans la dernière partie.

Quel sens donner enfin à l'appropriation féministe de l'herméneutique? Loin de se limiter à l'excavation de ressources de la tradition utiles pour le féminisme (même quand cette excavation est accompagnée d'une relecture critique ${ }^{24}$ ), une telle appropriation demande en fait un nouveau commencement. Celui-ci ne signifie pas le rejet pur et simple (flat-out rejection, Witt \& Shapiro 2014) de l'herméneutique «canonique »: car la position de toute herméneutique, orientée vers les valeurs de communication et de médiation, serait en quelque sorte d'envisager d'emblée la possibilité d'un dialogue avec la tradition. Ce commencement ne pourrait alors prendre forme qu'en associant, d'une part, une «herméneutique du soupçon» (Ricœur 1969) envers la tradition, et de l'autre, la reconnaissance et l'explicitation de la dimension herméneutique du féminisme. La seule question de l'usage de l'herméneutique traditionnelle par les féministes ne saura ici conduire au projet d'herméneutique féministe. En effet, penser l'herméneutique féministe ne revient pas à se demander ce que les féministes pourraient trouver utile dans la théorie herméneutique, ni à "sauver» (au sens dont en parle Elam) l'herméneutique traditionnelle en corrigeant ses présupposés androcentristes, mais exige de resituer la question herméneutique au sein du féminisme lui-même. 


\section{Vers une herméneutique féministe : le pouvoir de donner sens et (in)justice herméneutique} chercheuses féministes ont souligné comme nécessité d'ordre herméneutique, à savoir la mise en œuvre de la logique du genre au sein de la production du sens. Tina Fernandes Botts évoque ainsi dans son analyse des tentatives d'autrices féministes (Alcoff 2006; Warnke 2008) d'avoir recours à l'herméneutique afin de situer les catégories de genre et de race comme les «systèmes d'intelligibilité au sein desquels a lieu la compréhension et la production du sens" (Botts 2014: 511). Mais le déni du genre découle dans la théorie herméneutique du refus de voir dans la compréhension et la production du sens un enjeu politique. Une herméneutique féministe aurait donc l'intérêt de souligner ce double enjeu dans le rapport entre la production du sens et le genre : premièrement, de mettre en avant la puissance interprétative de la catégorie du genre comprise en termes d'horizon de sens, de «lieu [...] à partir [duquel] chacune d'entre nous comprend et expérimente le monde » (ibid. : 511) - horizon dont on reste trop souvent inconsciente -, et, deuxièmement, de considérer le donner-sens comme élément essentiel d'une "vie féministe » (Ahmed 2017) et comme un outil de lutte et de transformation politique.

J'évoquerai ici brièvement deux pistes (parmi de nombreuses autres) afin de penser une herméneutique féministe. La première serait de réunir, sous le nom d'herméneutique, les approches interprétatives féministes et notamment les stratégies d'exégèse alternatives qui structurent les rapports de la pensée féministe à la tradition androcentriste ${ }^{25}$. Ces interprétations féministes de la tradition constituent un support critique massif pour la théorisation féministe. Ici, l'herméneutique féministe serait susceptible d'encadrer les différentes pratiques de retravail du sens constitué et transmis au sein de la tradition dominante, se situant à la fois dans la logique du " soupçon ", c'est-à-dire en tant qu'une herméneutique critique de la tradition, et dans une démarche créatrice de relecture radicale du canon, consciente de l'inscription du genre et d'autres rapports de domination dans notre compréhension de textes et de concepts. Une telle étude des pratiques féministes de l'interprétation est susceptible de donner une meilleure compréhension de soi au féminisme. Elle pourrait en outre fournir une réflexion sur ce que peut être «la» tradition féministe (ou les traditions féministes), avec son héritage textuel, théorique, conceptuel, son histoire et son historicité, et en s'interrogeant plus particulièrement sur la constitution d'une communauté féministe à travers les textes et les lectures féministes ${ }^{26}$. Les questions de la transmission, de la citation et de la mémoire féministes entrent également dans le champ d'interrogation de l'herméneutique féministe.

La seconde piste demande donc d'élargir l'approche herméneutique entendue uniquement comme interprétation de textes ou du canon de pensée, afin de poser l'herméneutique et l'interprétation comme un rapport théorique et pratique au monde, qui peut être formulé en termes d'être-au-monde et d'engagement politique féministes. En ce sens plus large, c'est la démarche féministe elle-même qui deviendrait une démarche herméneutique : non seulement dans son travail de transformation du sens qui est donné aux rapports et normes sociales, mais en tant qu'elle agit pour articuler en mots des expériences et des injustices jusque-là non-prononcées, et ainsi inaudibles, donc absentes de l'horizon du comprendre collectif. Cet aspect révélateur du féminisme 
est souligné par Susanne de Lotbinière-Harwood : «En proposant une grille de lecture autre, c'est-à-dire genrée au féminin, le féminisme permet de voir et d'articuler des réalités cachées par et dans les mots.» (1991: 19, cité par Wilhelm 2014 : 159). Souvent réduite à son aspect d'être-vers-le-texte, l'herméneutique agit également là où aucun « texte » n'a été produit ou transmis - elle est l'élaboration même de cette production ou transmission. Ainsi, le travail féministe de l'explicitation et de l'interprétation ne sert pas seulement à donner une nouvelle explication à ce qui est déjà articulé dans la compréhension commune - mais œuvre à élucider quelque chose qui n'a pas de nom, une expérience qui, bien que présente et ressentie collectivement, n'accède pas à la parole et donc à la sphère de l'intelligible.

On trouverait un exemple d'un tel travail dans un essai de l'écrivaine états-unienne Rebecca Solnit (2018) où elle met en mots le phénomène des "Hommes qui nous Expliquent la Vie », ayant inspiré l'apparition du terme mansplaining (" mecsplication ») dans la foulée. Dans son essai, Solnit décrit notamment son expérience, longtemps sans nom, de conversations dans lesquelles des hommes, sans être experts en la matière, se mettaient avec beaucoup d'assurance à lui expliquer des choses dont elle possédait quant à elle une connaissance affinée, ce qui produisait l'effet de silence forcé ou de coupure de parole intimidante. Cette expérience est loin d'être isolée: "Toutes les femmes savent de quoi je parle » (Solnit 2018 : 14). La circulation étonnante de ce texte, sur laquelle Solnit revient dans le post-scriptum, montre qu'elle a réussi à mettre des mots sur quelque chose qui était ressenti et partagé par un grand nombre de femmes, mais qui se trouvait en quelque sorte dans un «vide» herméneutique («lacune » ou «trou », gap, comme l'appelle Miranda Fricker), à défaut d'être nommé ou décrit.

L'idée d'un vide herméneutique est aussi mobilisée par la philosophe allemande Carolin Emcke (2019) dans le contexte de sa réflexion sur \#MeToo. Dans son récit mettant au jour l'histoire d'une amie qui subissait des violences conjugales et le mur de silence qui l'entourait, Emcke emploie des termes comme résistance à la compréhension ou incompréhension afin de pointer un problème qui demande une prise en charge épistémologique:

C'est ce qui explique que le problème posé par les abus et les crimes n'est pas seulement moral, mais aussi cognitif: ils résistent à la compréhension, car ils ne peuvent coïncider avec les attentes qui nous ont été inculquées face au monde et aux autres. Ils font forcément naître de l'incompréhension. (p. 80)

Cette non-intelligibilité des expériences de la violence, de l'oppression et de la marginalisation a été conceptualisée dans des travaux de Fricker (2007) et de Laura Beeby (2011) en termes d'injustice herméneutique - concept qui, avec celui de justice herméneutique, contribue à mettre en lumière les enjeux politiques et féministes de la compréhension et de la production du sens, comme fondement du projet d'herméneutique féministe.

Selon la définition de Fricker, l'injustice herméneutique (qui est une forme d'injustice épistémique ${ }^{27}$ ) désigne le fait « d'avoir une partie importante de son expérience sociale occultée de la compréhension collective en raison d'un préjugé identitaire structurel dans la ressource herméneutique collective » $(2007: 155)$, une occultation qui affecte la capacité de l'individu à donner un sens à ses expériences sociales. Fricker inscrit l'injustice herméneutique dans un contexte plus large de la «marginalisation herméneutique " (ibid. : 153), celle-ci marque l'exclusion du champ de partage des significations sociales voire des pratiques mêmes à travers lesquelles sont générées ces 
significations. Pour donner un exemple paradigmatique de l'injustice herméneutique, elle s'appuie sur l'histoire de Carmita Wood ${ }^{28}$, qui a contribué à la genèse du concept de harcèlement sexuel. Beeby, qui reprend de manière critique l'analyse de Fricker, propose de penser comme une injustice herméneutique le moment exact où Wood se trouve dans l'impossibilité de nommer l'expérience du harcèlement sexuel qu'elle a subi de la part de son supérieur, dans la mesure où cette impossibilité tient au fait que " cette expérience, celle que nous entendons maintenant comme harcèlement sexuel, a été "occultée de la compréhension collective" » (2011 : 480). L'injustice herméneutique ne se confond pas avec l'expérience de l'agression elle-même, elle souligne un préjudice particulier causé par l'expérience « d'être incapable de comprendre et de communiquer sur le harcèlement sexuel » (ibid.: 481). Enfin, le moyen de surpasser cette injustice a été pour Wood la découverte du caractère commun de cette expérience partagée avec d'autres femmes au sein d'un groupe de parole féministe et finalement l'élaboration collective du concept de sexual harassment.

Cette élaboration conceptuelle rentre dans la logique de ce qu'on pourrait appeler la justice herméneutique. Sans engager davantage ici une réflexion sur les réparations possibles des injustices herméneutiques, je tenterai d'élargir la définition de ce concept que donne Fricker - une "vertu » particulière de la réceptivité aux paroles d'autrui, aussi bien qu'à ses silences $(2007: 168)$ - pour désigner la justice herméneutique comme résultat d'un travail d'explicitation et de mise en mots des expériences encore sans nom qui permet aussi de communiquer leurs significations aux autres, et en fin de compte de forger des outils conceptuels puissants mobilisant l'action collective.

\section{Conclusion}

41 La lutte féministe ne prendrait-elle pas, parmi ses nombreuses déclinaisons, celle d'une lutte pour l'inclusion dans le comprendre collectif et pour la participation à la production du sens ? C'est à partir de là qu'on pourrait penser l'importance et la pertinence du projet d'une appropriation féministe de l'herméneutique esquissé dans le présent article. Or cette appropriation serait en fait aussi une réappropriation ${ }^{29}$ : elle permettrait au féminisme de se (re)connaître comme une herméneutique, en libérant de leur monopolisation androcentriste et de leur théorisation dépolitisée (et ainsi en se réappropriant) les questions de la production, de l'interprétation et de la transmission du sens, en se faisant ainsi une place dans des espaces où les femmes ont été invisibilisées depuis des siècles ou qui se sont construits sans elles. Ce faisant, l'herméneutique féministe doit encore parcourir le chemin sur lequel, de son côté, la traductologie féministe aurait déjà bien avancé ${ }^{30}:$ réinvestir et remodeler ces univers andro- et eurocentristes en revendiquant des enjeux politiques là où ils ont longtemps été niés.

Dans la mesure où interpréter le monde ne signifie pas nécessairement le transformer, la dimension politique de l'herméneutique n'est pas évidente, et un passage peut sembler nécessaire entre le registre de l'interprétation et celui de la réalité sociale qui "se passerait" parfois en dehors du domaine des signes. Ainsi, les expériences du sexisme et du racisme seraient des non-signes, et de ce fait rangées hors le registre herméneutique, ou interprétatif. Les outils de l'herméneutique sont-ils appropriés là où il n'y a encore aucun signe à interpréter? Dans mon travail sur l'herméneutique féministe, j'ai voulu dépasser la vision de l'herméneutique comme une théorie de l'interprétation des signes uniquement, car cette approche me semblait réductrice. Au 
lieu de construire là encore une herméneutique explicative, il m'a paru plus pertinent d'imaginer une herméneutique créatrice, ayant trait à l'agentivité et à la performativité, rendant possible une action sur le monde. Dans la mesure où le féminisme porte en lui cet élan de dépassement, de retournement, de déconstruction des significations existantes, une herméneutique féministe ne saurait se limiter à la simple interprétation de celles-ci. Mon approche consiste donc à ne pas cantonner l'herméneutique à la seule sphère du "déjà-intelligible ", mais de l'inclure partout où il y a élaboration du sens à partir du non-sens, constitutive du rapport féministe au monde et de mettre ainsi en lumière le lien entre donner-sens et agir politique qui constitue un point encore peu développé dans le cadre des recherches féministes et mérite une prise en compte et une étude plus conséquentes.

En resituant la production et la transmission du sens au sein de la praxis féministe, l'herméneutique féministe peut fournir des ressources aussi bien de survie, que d'élaboration créatrice du sens, comme on peut le lire à partir de cette description du féminisme proposée par Sara Ahmed, comme processus de donner un sens (sense-making process, Ahmed $2017: 20)$ à ce qui, comme les expériences du sexisme et du racisme, relève du « non-sens » : « Féminisme : comment nous survivons aux conséquences de ce à quoi nous sommes confrontés en offrant de nouvelles façons de comprendre ce à quoi nous nous confrontons.» (p.22, je souligne). Ainsi, nous complétons la «ressource herméneutique collective » et contribuons à un monde plus juste.

\section{BIBLIOGRAPHIE}

AHMED, Sara. 2017. Living a Feminist Life. Durham/London : Duke University Press.

ALCOFF, Linda Martín. 2003. « Gadamer's Feminist Epistemology » in Feminist Interpretations of Hans-Georg Gadamer, CODE Lorraine (éd.). University Park, PA : The Pennsylvania State University Press, 231-258.

ALCOFF, Linda Martín. 2006. Visible Identities : Race, Gender, and the Self. Oxford : Oxford University Press.

BARLAS, Asma. 2002. « Believing Women » in Islam : Unreading Patriarchal Interpretations of the Qur'an. Austin : University of Texas Press.

BEEBY, Laura. 2011. « A Critique of Hermeneutical Injustice » Proceedings of the Aristotelian society $111(3)$ : 479-486.

BORDO, Susan. 1987. The Flight to Objectivity: Essays on Cartesianism and Culture. Albany : State University of New York Press.

BOTTS, Tina Fernandes. 2014. « Hermeneutics, Race, and Gender » in The Routledge Companion to Hermeneutics, MALPAS, Jeff \& GANDER, Hans-Helmuth (éds.). London/New York : Routledge, 498-518.

BOWLES, Gloria. 1984. « The Uses of Hermeneutics For Feminist Scholarship » Women's Studies International Forum 7 : 185-188. 
BRIÈRE, Laurence, LIEUTENANT-GOSSELIN Mélissa \& PIRON Florence (éds.). 2018. Et si la recherche scientifique ne pouvait pas être neutre? Québec : Éditions science et bien commun.

BROWNMILLER, Susan. 1990. In Our Time : Memoir of a Revolution. New York : Dial Press.

BRUGÈRE, Fabienne. 2014. Qui a peur des philosophes ? Paris : Bayard.

BUKER, Eloise. 1990. « Feminist Social Theory and Hermeneutics : An Empowering Dialectics?» Social Epistemology 4(1) : 23-39.

CAPUTO, John D. 1987. Radical Hermeneutics. Repetition, Deconstruction, and the Hermeneutic Project. Bloomington : Indiana University Press.

CODE, Lorraine. 2003. «Why Feminists Do Not Read Gadamer » in Feminist Interpretations of HansGeorg Gadamer, CODE Lorraine (éd.). University Park, PA : The Pennsylvania State University Press, 1-36.

CODE, Lorraine (éd.). 2003. Feminist Interpretations of Hans-Georg Gadamer. University Park, PA : The Pennsylvania State University Press.

COULOMB-GULLY, Marlène. 2009. «Les sciences de l'information et de la communication : une discipline Gender blind? » Questions de communication 15(1) : 129-153.

COULOMB-GULLY, Marlène \& RENNES, Juliette. 2010. « Genre, politique et analyse du discours. Une tradition épistémologique française gender blind » Mots. 30 ans d'études des langages du politique (1980-2010) $94: 175-182$.

DERRIDA, Jacques. 1978. Éperons : les styles de Nietzsche. Paris : Flammarion.

ELAM, Diane. 1991. «Is Feminism the Saving Grace of Hermeneutics? » Social Epistemology 5(4) : 349-360.

EMCKE, Carolin. 2019. Quand je dis oui... Traduit par Alexande Pateau. Paris : Seuil.

FIORENZA, Elisabeth Schüssler. 1986 [1983]. En mémoire d'elle. Essai de reconstruction des origines chrétiennes selon la théologie féministe. Traduit par Marcelline Brun. Paris : Éditions du Cerf.

FLEMING, Maria. 2003. « Gadamer's Conversation : Does the Other Have a Say? » in Feminist Interpretations of Hans-Georg Gadamer, CODE Lorraine (éd.). University Park, PA : The Pennsylvania State University Press, 109-132.

FRICKER, Miranda. 2007. Epistemic Injustice: Power and Ethics of Knowing. Oxford : Oxford University Press.

GADAMER, Hans-Georg. 1982. «Rhétorique, herméneutique et critique des idéologies. Commentaires métacritiques de Vérité et méthode » in L'Art de comprendre. Écrits I: Herméneutique et tradition philosophique. Traduit par Marianna Simon. Paris : Aubier-Montaigne, 123-144.

GADAMER, Hans-Georg. 1996. Vérité et méthode : les grandes lignes de l'herméneutique philosophique. Traduit par Pierre Fruchon et al. Paris : Seuil.

GADAMER, Hans-Georg. 2004. «L'incompétence politique de la philosophie » in Esquisses herméneutiques : essais et conférences. Traduit par Jean Grondin. Paris : Vrin, 53-60.

GAUTIER, Claude. 2018. «De la neutralité axiologique au réalisme des expériences vécues du standpoint. Une critique féministe de la relation de connaissance » in Épistémologies du genre : croisements des disciplines, intersections des rapports de domination, GenERe (éd.). Lyon : ENS Éditions, 97-115. 
HAMIDI, Malika. 2015. «La pensée féministe islamique à l'ère de la mondialisation : entre stratégie herméneutique et mobilisation transnationale » L'Homme \& la Société 4(198) : 113-125.

HARTSOCK, Nancy. 2004. « The Feminist Standpoint : Developing the Ground for a Specifically Feminist Historical Materialism », in The Feminist Standpoint Theory Reader. Intellectual \& Political Controversies, HARDING Sandra (éd.). New York \& Londres : Routledge, 35-53.

LAMRABET, Asma. 2007. Le Coran et les femmes : une lecture de libération. Lyon : Tawhid.

LOTBINIÈRE-HARWOOD, Susanne de. 1991. Re-belle et infidèle. La traduction comme pratique de réécriture au féminin. The Body Bilingual, Translation as a Rewriting in the Feminine. Montréal : Les éditions du remue-ménage/Women's Press.

PARMENTIER, Elisabeth. 1999. Les filles prodigues : défis des théologies féministes. Genève : Labor et Fides.

PARMENTIER, Elisabeth, DAVIAUT Pierette \& SAVOY Lauriane (éds.). 2018. Une bible des femmes : vingt théologiennes relisent des textes controversés. Genève : Labor et Fides.

PAVEAU, Marie-Anne. 2018. «Le genre : une épistémologie contributive pour l'analyse du discours » in Épistémologies du genre : croisements des disciplines, intersections des rapports de domination, GenERe (éd.). Lyon : ENS Éditions, 79-95.

PLAZA, Monique. 1978. « Nos dommages et leurs intérêts » Questions Féministes 3 : 93-103.

PLAZA, Monique. 1984. « Ideology Against Women » Feminist Issues 4(1) : 73-82

« Réappropriations - Paradoxes des stratégies féministes d'émancipation ». 2019. Appel à contribution [En ligne], Calenda, publié le 16 décembre 2019, consulté le 14 septembre 2020. URL : https://calenda.org/720152

RICCEUR, Paul. 1969. Le conflit des interprétations : essais d'herméneutique. Paris : Seuil.

ROONEY, Phyllis. 1991. « Gendered Reason : Sex, Metaphor and Conceptions of Reason » Hypatia 6(2) : 77-103.

SOLNIT, Rebecca. 2018. Ces hommes qui m'expliquent la vie. Traduit par Céline Leroy. Paris : Éditions de l'olivier.

STANTON, Elizabeth Cady. 2002 [1895]. The Woman's Bible : A Classic Feminist Perspective. Mineola, NY : Dover Publications.

WADUD, Amina. 1999. Qur'an and Woman : Rereading the Sacred Text from a Woman's Perspective, 2nd Edition. Oxford : Oxford University Press.

WARNKE, Georgia. 1993. « Feminism and Hermeneutics » Hypatia 8(1) : 81-98.

WARNKE, Georgia. 2008. After Identity : Rethinking Race, Sex, and Gender. Cambridge : Cambridge University Press.

WILHELM, Jane. 2014. « Anthropologie des lectures féministes de la traduction » TTR : traduction, terminologie, rédaction 27(1) : 149-188.

WITT, Charlotte \& SHAPIRO, Lisa. 2014 [2000]. « Feminist History of Philosophy » in The Stanford Encyclopedia of Philosophy (Fall 2018 Edition) [En ligne], ZALTA Edward N. (éd.). Consulté le 1 octobre 2019. URL : https://plato.stanford.edu/archives/fall2014/entries/feminism-femhist/ 


\section{NOTES}

1. Ce texte est issu d'une recherche post-doctorale indépendante et auto-financée que j'ai menée entre 2018 et 2020. Je remercie les coordinatrices de FELiCiTE d'avoir encouragé et accueilli ce projet d'abord sous forme de conférence présentée à l'ENS de Lyon en juin 2019 dans le cadre d'un séminaire de traductologie féministe. Tous mes remerciements vont aussi à Jane Wilhelm avec qui j'ai pu avoir de précieux échanges au sujet de l'herméneutique et du féminisme lors de son intervention pour FELiCiTE le 25 janvier 2019.

2. Il s'agit de l'épistémologie que Laurence Brière, Mélissa Lieutenant-Gosselin et Florence Piron, dans leur introduction au collectif Et si la recherche scientifique ne pouvait pas être neutre?, désignent comme « le modèle dominant (positiviste réaliste) » « dans le régime contemporain de développement des savoirs savants" (Brière et al. $2018: 1$ ) : une épistémologie (relevant notamment du domaine des SHSL) qui privilégie le modèle des sciences dites exactes et promeut le rationalisme, voire le scientisme, la validité scientifique réservée aux «faits " vérifiés par l'expérience, ainsi que la neutralité et l'impartialité supposées du chercheur/de la chercheuse face à son objet d'étude (objectivisme).

3. Gender blindness, expression anglaise par analogie avec color blindness (le fait de ne pas faire attention à la couleur de la peau), désigne une approche qui se caractérise par l'ignorance des différences de genre et la non-prise en compte des inégalités existantes entre les sexes. En France, des chercheuses (Marlène Coulomb-Gully, Juliette Rennes, Marie-Anne Paveau) ont souligné le caractère gender blind des disciplines comme les sciences de l'information et de la communication ou l'analyse du discours (voir Coulomb-Gully 2009, Coulomb-Gully \& Rennes 2010, Paveau 2018).

4. Appropriation, mot qui possède parfois une connotation négative dans des contextes féministes, comme récupération (de luttes) ou possession (du corps des femmes), peut désigner au contraire une démarche subversive. Je l'emploie ici (et dans le reste de l'article) à la fois au sens d'une stratégie interprétative féministe décrite par Charlotte Witt et Lisa Shapiro (2014 [2000]) - l'usage de concepts et théories non-féministes à des fins féministes - et au sens d'une prise de pouvoir créatrice qui consiste à envahir des espaces d'où on a été exclu'e et les investir d'un sens nouveau.

5. Sur la question du « réalisme étendu » de la perspective féministe, notamment à partir des épistémologies du standpoint, voir Gautier 2018.

6. https://felicite.hypotheses.org/a-propos

7. Néologisme dont l'invention au début des années 1970 est attribuée à Robin Morgan - autrice, artiste et militante féministe -, le terme herstory par son jeu de mots en anglais désigne une histoire écrite d'un point de vue féministe et mettant en avant les vies et les réalisations des femmes, négligées ou occultées dans l'histoire hégémonique dont l'écriture a été monopolisée par les hommes blancs (his-tory).

8. L'utilisation du masculin renvoie ici à la nette prépondérance des hommes parmi les théoriciens du courant.

9. Quoique ces pratiques commencent bien avant: un des exemples en est The Woman's Bible, éditée aux États-Unis par Elizabeth Cady Stanton entre 1895 et 1898 (Stanton 2002 [1895]). Parmi les théologiennes féministes chrétiennes qui ont inclus l'herméneutique dans leur travail, on pourrait mentionner Elisabeth Schüssler Fiorenza (1986 [1983]) ou encore Elisabeth Parmentier (1999) qui a co-dirigé récemment, en 2018, avec Pierrette Daviau et Lauriane Savoy, Une bible des femmes (qui fait écho à The Woman's Bible). On trouve également de nombreux usages de l'approche herméneutique en théologie féministe islamique, dans les travaux de Asma Barlas (2002), Malika Hamidi (2015), Asma Lamrabet (2007), Amina Wadud (1999). Des « herméneutiques féministes " judaïques ont été mobilisées au sein des écrits de Judith Plaskow, Elyse Goldstein, etc. 
10. Les deux points ont été examinés de manière plus détaillée et dans le contexte de l'herméneutique gadamérienne par Lorraine Code dans son texte « Why Feminists Do Not Read Gadamer » (2003).

11. Voir par exemple son essai de 1992-1993, "L'incompétence politique de la philosophie » (Gadamer 2004).

12. Warnke (1993) inclut également dans la tradition herméneutique les auteurs comme Michael Walzer, Alistair McIntyre, John Rawls et Charles Taylor, qu'elle place notamment dans le courant d'« herméneutique politique ».

13. Ici : terme à visée critique qui met en évidence le caractère dominant de la raison et du langage (logos) dans la pensée occidentale.

14. Le cas de l'herméneutique est en ceci intéressant qu'elle occupe ici une position ambiguë, en critiquant la posture du positivisme, en convergence avec les critiques proposées par les épistémologies féministes (bien que dans un cadre théorique totalement différent), sans pour autant remettre en question ses propres présupposés androcentristes. Cette ambigüité peut être instructive en cela que la déconstruction de la rationalité objectiviste est une condition insuffisante pour la naissance d'une herméneutique féministe.

15. Traduction par mes soins. Sauf mention contraire, cela concerne toutes les citations traduites de l'anglais dans l'ensemble de l'article.

16. Elle l'explique comme « les dangers de l'usage abusif de notre pensée par les hommes qui ont plus de pouvoir dans le monde des idées parce qu'ils sont des hommes » (p. 186-187).

17. «Je suggérerais donc que l'une de nos nombreuses stratégies soit l'utilisation de ces traditions hétérodoxes, puisque pour les chercheuses et chercheurs qui ne font pas actuellement partie de nos cercles, ces critiques pourraient être un prélude à une compréhension de la pensée féministe. $\gg($ p. 187)

18. Les cas d'Althusser et de Foucault ont été analysés par Monique Plaza dans deux articles devenus classiques, respectivement de 1984 («Ideology Against Women») et de 1978 («Nos dommages et leurs intérêts »).

19. Dans la théorie herméneutique, ce concept se réfère à l'opération de l'application d'un texte à la situation présente. L'exemple privilégié en est la prédication du texte biblique.

20. Les deux textes ont été publiés dans des numéros successifs de la revue Social Epistemology en 1990 et en 1991.

21. Non seulement elle renvoie à la lecture d'un texte difficile d'accès même pour des spécialistes, mais elle ne semble pas non plus aussi radicale puisqu'elle invite à se servir encore d'une théorie élaborée par un homme et dont les présupposés sexistes propres n'ont pas été questionnés, dont l'approche reste globalement celle de la «masculinité abstraite » (Hartsock 2004 : 44).

22. «Synoptique» signifie que la tradition philosophique occidentale est prise par la critique féministe comme un tout, et que c'est en tant que telle qu'elle apparaît comme genrée au masculin (male gendered) jusqu'à ses valeurs et normes les plus profondes (concepts de raison, d'objectivité, etc.). Cette approche se distingue des interprétations qui visent une critique féministe de concepts particuliers (ex. le concept de matière chez Aristote) sans que les théories dont relèvent ces concepts soient considérées comme intrinsèquement sexistes ou misogynes.

23. Sur les différents sens du caractère masculin de la raison dans l'histoire de la philosophie, où la «masculinité » peut être symbolique/métaphorique ou sociale et culturelle, voir Witt \& Shapiro (2014), ainsi que Bordo (1987) et Rooney (1991).

24. «Les féministes qui considèrent le canon philosophique comme une ressource à exploiter pour trouver des idées utiles au féminisme sont engagées dans une activité potentiellement oppressive puisque l'histoire de la philosophie regorge d'idées et de théories qui sont (ou pourraient être) oppressantes pour les femmes aujourd'hui. Le défaut épistémique est que 
l'approche de l'appropriation est trop révérencieuse de la tradition et pourrait donc ne pas être suffisamment critique à son égard. » (Witt \& Shapiro 2014)

25. À ce propos, il serait intéressant de se rapporter à la collection « Re-reading the Canon » de Pennsylvania State University Press (éditée par Nancy Tuana) qui réunit les interprétations féministes d'auteur'trice's « canoniques ».

26. Ainsi, par exemple, Sara Ahmed suggère une idée de corpus féministe entendu en termes de «classiques féministes» (feminist classics) ou «textes de compagnie » (companion texts, par analogie avec companion species de Donna Haraway). Pour elle, la notion de classiques féministes ne signifie pas la constitution d'un canon féministe (alternatif au canon androcentriste), mais invite à penser « comment les livres font communautés » (Ahmed $2017: 17$ ).

27. Comme l'explique Fricker, les injustices épistémiques ne doivent pas être comprises au sens d'une justice « distributive », mais concernent spécifiquement les capacités de l'individu comme sujet de connaissance (" as a knower») (2007:1).

28. Harcelée sexuellement par son supérieur, Carmita Wood, employée administrative de la North American University dans les années 1970, finit par quitter son poste. Au moment de formuler la raison de sa démission pour la demande de l'assurance chômage, Wood ne parvient pas à mettre des mots sur son expérience, puisque l'époque ne connaît pas la notion de harcèlement sexuel. L'histoire de Wood a été retranscrite par Susan Brownmiller (1990 : 280-281). 29. En complétant le terme d'«appropriation" par celui de réappropriation (tout aussi polémique), je souhaite éviter chez le·a lecteurtrice l'impression que le champ herméneutique serait un champ complètement étranger aux femmes qu'il s'agirait d'occuper (de s'approprier) pour la première fois. Ré- ici renvoie à la présence et à la participation (systématiquement invisibilisées et dévalorisées) des femmes à la construction des savoirs, que l'herméneutique féministe aurait justement vocation à récupérer et à ré-valoriser (via diverses stratégies interprétatives). Cette ré-appropriation (féministe) serait ainsi la réponse à l'appropriation (masculine) de l'espace intellectuel ou de l'horizon herméneutique dominant. N'oublions pas les concepts comme "retournement» (ou encore reclaim) qui pourraient éclairer un tel usage du terme de réappropriation, mis en avant notamment dans ce récent appel à contribution : « Réappropriations - Paradoxes des stratégies féministes d'émancipation » (https://calenda.org/ 720152).

30. Avec les travaux de Gayatri Spivak, Luise Von Flotow, Susanne de Lotbinière-Harwood, Lori Chamberlain, Sherry Simon, Rosemary Arrojo et d'autres.

\section{RÉSUMÉS}

Qu'est-ce qu'interpréter en féministe? Comment s'approprier féministement des textes, des théories et des concepts qui relèvent du canon et sont porteurs de la perspective mâle blanche? Est-ce qu'une interprétation peut être « juste » sans tenir compte des dynamiques de pouvoir qui agencent la production et la transmission du sens? Cet article s'inspire de ces questions en partant d'un territoire peu fréquenté par les féministes : celui de l'herméneutique. La question se pose alors de savoir dans quelle mesure l'approche herméneutique pourrait dialoguer avec le féminisme, voire faire l'objet d'une appropriation féministe. L'article revient d'abord sur quelques interprétations que des autrices féministes ont consacrées à la pensée herméneutique dans les années 1980-1990, afin de s'interroger ensuite sur les enjeux et la pertinence d'une herméneutique féministe. Une telle recherche au croisement de l'herméneutique et du 
féminisme pourrait contribuer à mieux situer sinon à élargir le champ de la traductologie féministe, dont l'herméneutique féministe serait une théorie alliée.

What is feminist interpretation? How to feministically grasp canonical texts, theories and concepts, all of them emerging from pale male perspective? Can an interpretation be "right" without taking into account the power dynamics that shape the production and transmission of meaning? The paper draws on these questions by focusing on a territory that feminists rarely navigate: that of hermeneutics. The question thus arises as to the extent to which the hermeneutic approach could enter into dialogue with feminism, or even be appropriated by feminists. The paper first reviews some of the feminist interpretations of hermeneutics from the 1980s-1990s, in order to consider then the issues at stake and the relevance of feminist hermeneutics. Such research at the crossroads of hermeneutics and feminism could contribute to better situate, if not widen, the boundaries of feminist traductology, of which feminist hermeneutics would be an allied approach.

INDEX

Thèmes : Recherches

Keywords : feminism, hermeneutics, epistemology, interpretation, hermeneutical justice

Mots-clés : féminisme, herméneutique, épistémologie, interprétation, justice herméneutique

\section{AUTEUR}

\section{AXELLE CRESSENS}

Axelle Cressens est docteure en philosophie, chercheure indépendante basée à Lyon. Elle est l'auteure d'une thèse intitulée « Figures de la temporalité dans l'herméneutique de H.-G.

Gadamer ». Membre associée du Centre de recherches interdisciplinaires en sciences humaines et sociales (CRISES, Montpellier), elle est coresponsable (avec Yosra Ghliss) du projet GRASS Groupe de réflexion autour des savoirs situés. Son travail se situe au croisement de l'herméneutique et des recherches féministes et porte essentiellement sur les problématiques épistémologiques qui émergent à l'intersection de ces deux champs. Parmi ses centres d'intérêt, les éthiques de la vulnérabilité ou encore la lesbian theory. Avec Aurélie Knüfer, elle a codirigé un dossier thématique « Mobilisations de vulnérabilité » à paraitre prochainement dans la revue Genre, sexualité \& société. 